\title{
A Novel Approach for the Detection of Drunken Driving using the Power Spectral Density Analysis of EEG
}

\author{
E.Malar \\ Dept. of Biomedical Engg, \\ PSG college of technology \\ Coimbatore-04 \\ Tamilnadu, India
}

\author{
M.Gauthaam \\ Dept. of Biomedical Engg, \\ PSG college of technology \\ Coimbatore-04 \\ Tamilnadu, India
}

\author{
D.Chakravarthy \\ Dept. of Biomedical Engg, \\ PSG college of technology \\ Coimbatore-04 \\ Tamilnadu, India
}

\begin{abstract}
In today's scenario, more and more people are required to travel back and forth to various places. With the increasing vehicular population and their movements on the roads, accidents are also steadily increasing. It has become a nightmare for the authorities to prevent /reduce such fatal accidents on the roads. But the authorities' efforts are in vain. It is shocking to know the study results that around $50 \%$ of the road accidents are owing to drunken driving all over the world $^{[1][2]}$. Any mechanism or device to reduce such deaths will be of great help. Drunken driving and its subsequent catastrophe can be avoided by monitoring the EEG of the driver. The power of the EEG signal in frontal region decreases with the increase in the amount of alcohol intake, and the power of the EEG signal in central, occipital region increases. Therefore, power spectral density can be used as a parameter to differentiate EEG of alcoholic from nonalcoholic, thereby reducing drunken driving. Results are presented to support this approach.
\end{abstract}

\section{General Terms: Signal Processing}

Keywords: Electroencephalography, alpha wave, theta wave, power spectral density.

\section{INTRODUCTION}

The proposed technology captures brain waves and stops drivers from causing accidents due to drunken driving. It is based on the fact that, alcohol affects the neuronal-activity of the brain ${ }^{[8], ~[9] ~ i . e ., ~ a l p h a ~ a c t i v i t y ~ o f ~ a l c o h o l i c ~ p e r s o n ~}$ decreases and theta activity increases (alpha and theta activity are various frequency bands of brain activity). The smart cap consists of five embedded electrode in the form of forehead band, which is used to acquire the EEG signal. The acquired EEG signal is pre-processed and directed via Bluetooth to the intelligence unit which consists of microprocessor. This algorithm loaded processor is used to decompose the EEG signal into alpha, beta, gamma and delta waves. The decomposed EEG signal is analysed for alcoholic activity. The voltage produced by the algorithm is used to drive the relay system depending upon the presence or absence of abnormalities in the EEG. The key hole of car engine is replaced by the relay system. Thus the engine will start only if there is no significance of abnormalities in the decomposed EEG signal.

\section{FLAWS IN CONVENTIONAL METHODS}

Breath analyser is the most common method of testing for blood-alcohol content in use today. Personal breath analyzers estimate the concentration of alcohol in the body by measuring the amount of alcohol exhaled from the lungs.
The existing 'Automated Breath Analyzer' for detecting alcohol, consumed by the driver, is no longer useful because of its own limitations \& demerits. This is based on the odour and the level of alcoholic $(-\mathrm{OH})$ ions present in the breath. Hence, it can easily be cheated if the suspect takes in spices or other smell attenuators. The other demerit is that sensors for detecting alcohol content in air within the vehicle ambience by using 'reducing (-OH reductases) or oxidizing enzymes ($\mathrm{OH}$ oxidases)' is ineffective because of its less sensitivity and accuracy. Also the enzymes used in the sensor have to be replaced periodically. Another method is using pulse oxymeters. Even though non invasive oxy-meters are available, a considerable amount of time is taken by the meters to detect $\mathrm{OH}$ ions (This is the time taken by alcoholic ions to reach the cells from blood stream). Thus this is also not reliable and lacks practical application as an automated system for detecting drunk driving.

\section{EEG}

Electroencephalography is the recording of electrical signals from the brain. Each nerve cell (neuron) in the brain produces a tiny electrical charge; when a number of neurons become active, the sum of these tiny electrical charges can be detected on the surface of the scalp. Small electrodes placed on the scalp detect this electrical activity, which is magnified and recorded as brain waves (neural oscillations). These brain waves illustrate the activity as it is taking place in various areas inside the brain. ${ }^{[23]}$

The rhythmic activity is divided into frequency bands:

Delta is the frequency range up to $4 \mathrm{~Hz}$. It tends to be the highest in amplitude and the slowest waves. It is seen normally in adults in slow wave sleep.

Theta is the frequency range from $4 \mathrm{~Hz}$ to $7 \mathrm{~Hz}$. Theta is seen normally in young children. It may be seen in drowsiness or arousal in older children and adults.

Alpha is the frequency range from $8 \mathrm{~Hz}$ to $12 \mathrm{~Hz}$. This is activity in the $8-12 \mathrm{~Hz}$ range seen in the posterior regions of the head on both sides, being higher in amplitude on the dominant side. It is brought out by closing the eyes and by relaxation.

Beta is the frequency range from $12 \mathrm{~Hz}$ to about $30 \mathrm{~Hz}$. It is seen usually on both sides in symmetrical distribution and is most evident frontally. Beta activity is closely linked to motor behavior and is generally attenuated during active movements. 


\section{DECOMPOSITION OF EEG USING STATIONARY WAVELET TRANSFORM}

In order to detect the presence of alcohol in EEG, it is necessary to analyse the temporal and spectral features of EEG ${ }^{[10]}$. The time-frequency analysis was carried using stationary wavelet transform. Stationary wavelet transform applies high and low pass filters to the data at each level without carrying out the process of decimation. Using stationary wavelet transform EEG was decomposed to 5 frequency levels (delta(0-4 hz), theta(4-8 hz), alpha(8-12 hz), beta(12-20 hz), gamma(20 hz and above)).

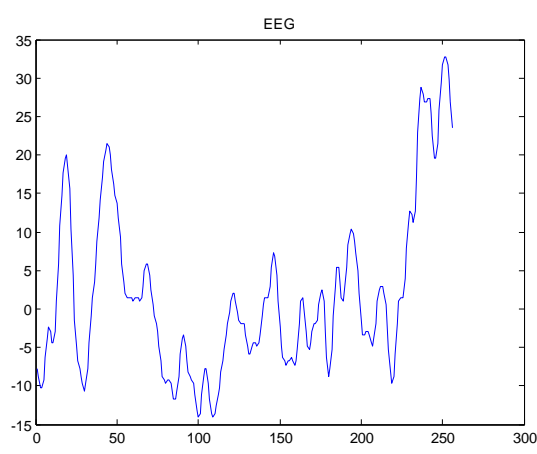

Fig 1: EEG wave

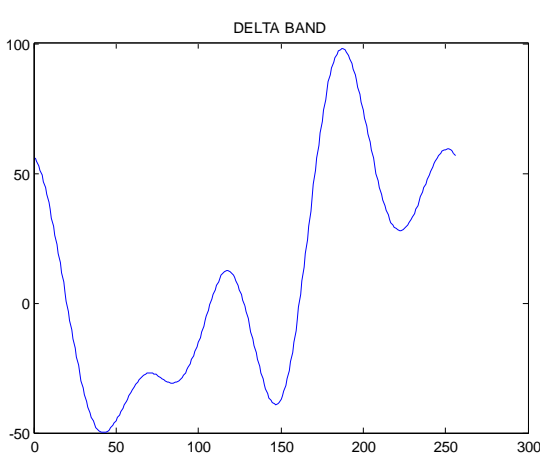

Fig 2: Delta band of EEG wave decomposed using stationary wavelet transform

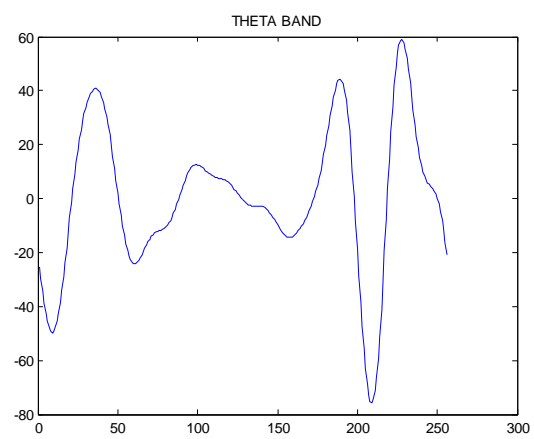

Fig 3: theta band of EEG wave decomposed using stationary wavelet transform

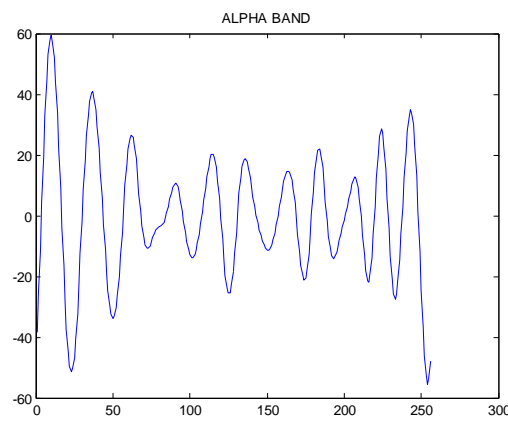

Fig 4: alpha band of EEG wave decomposed using stationary wavelet transform

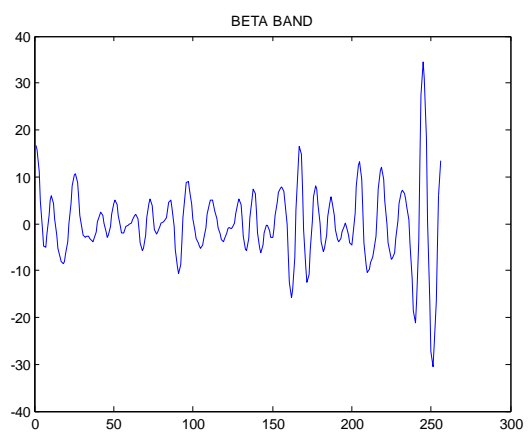

Fig 5: beta band of EEG wave decomposed using stationary wavelet transform

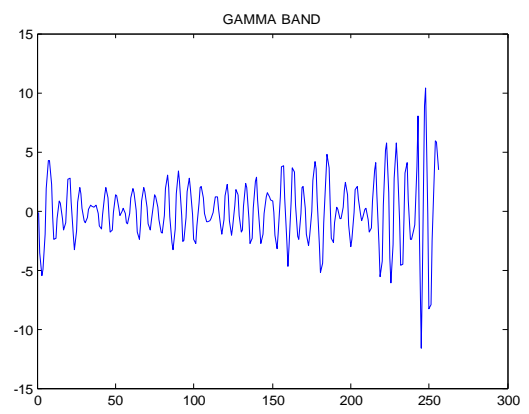

Fig 6: gamma band of EEG wave decomposed using stationary wavelet transform

\section{POWER SPECTRAL DENSITY} ANALYSIS

The Power Spectral Density analysis is performed for finding out the power of the signal over a particular frequency band [7].

The literature survey reveals that the power of the EEG signal in frontal region decreases with the increase in the amount of alcohol intake, and the power of the EEG signal in central, occipital region increases. This indicates that nerve stimulation of alcohol has a strong influence on central region, so people will become excited after drinking. ${ }^{[14],[20], ~[16] ~}$ 
1) $\operatorname{Theta}(\theta)$ waves:

$\operatorname{Theta}(\theta)$ waves begin to appear and gradually enhances after consuming alcohol as the subjects are driven in to the state of sleepy and the central nervous system of the subjects is inhibited. ${ }^{[5],[15]}$

\section{2) $\operatorname{Alpha}(\alpha)$ waves:}

Alpha ( $\boldsymbol{\alpha})$ waves gradually decreases and the region of alpha ( $\boldsymbol{\alpha})$ waves is expanded after consuming alcohol ${ }^{[5],[13]}$

\section{3) $\operatorname{Beta}(\beta)$ waves:}

The cerebral cortex remains in an excitable condition after consuming alcohol, which leads to beta( $\boldsymbol{\beta})$ waves gradually enhanced and the area of beta $(\boldsymbol{\beta})$ waves are expanded. ${ }^{[5],[17]}$

\section{RESULTS}

Based on the methodology discussed above, this section describes the results and their interpretation

EEG signals of 5 normal people and 5 alcoholic people were used and further analysis was carried out to find the correlation between EEG of normal person and alcoholic.

EEG signal was decomposed into 4 frequency bands (alpha, beta, delta and theta) and their power spectrum was analyzed and following results were obtained using MATLAB ${ }^{[11]}$

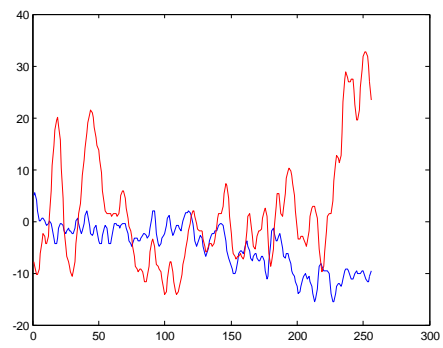

Fig 7: EEG of normal person (red) vs. alcoholic person (blue)

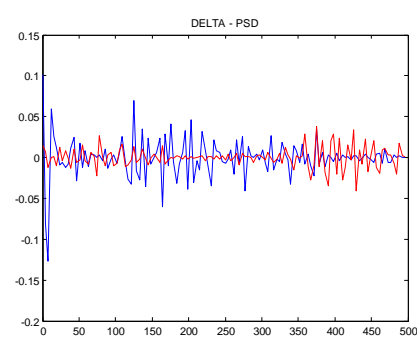

Fig 8: power spectrum density of delta wave of normal person (red) and alcoholic person (blue)

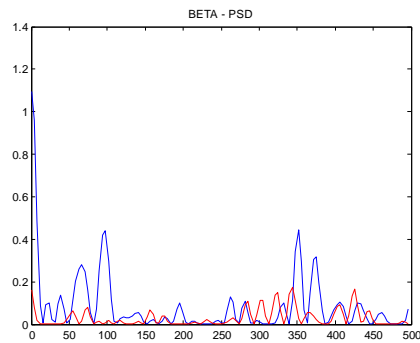

Fig 9: power spectrum density of beta wave of normal person (red) and alcoholic person (blue)

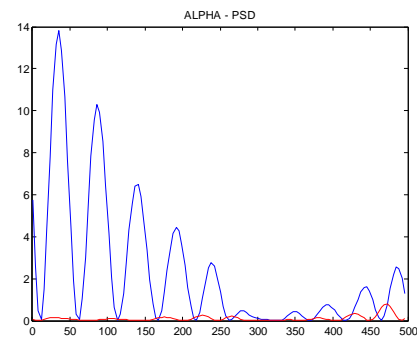

Fig 10: power spectrum density of alpha wave of normal person (red) and alcoholic person (blue)

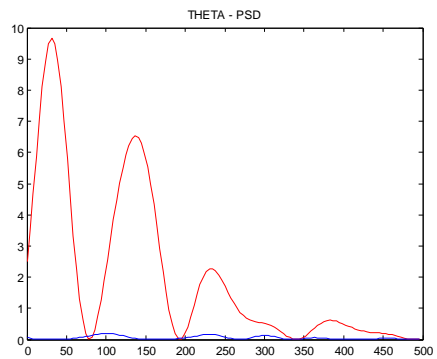

Fig 11: power spectrum density of theta wave of normal person (red) and alcoholic person (blue)

It is very much evident from the above plots that alpha activity is decreased in alcoholic person compared to normal person. Similarly theta activity is increased in alcoholic person compared to normal person. Going by these two facts normal person can be differentiated from alcoholics from their EEG.

\section{DISCUSSION}

From the above derived results, it is possible to devise a system to identify and prevent drunken driving. This technology will help in preventing such deaths (caused by drunken driving and drowsiness while driving) and it is a means to save thousands of priceless human lives ${ }^{[3]}$ [4]. This proposed system has assembly of 3 units namely

(i) Interface unit

(ii) Digital pre-processing unit

(iii) Intelligence unit 


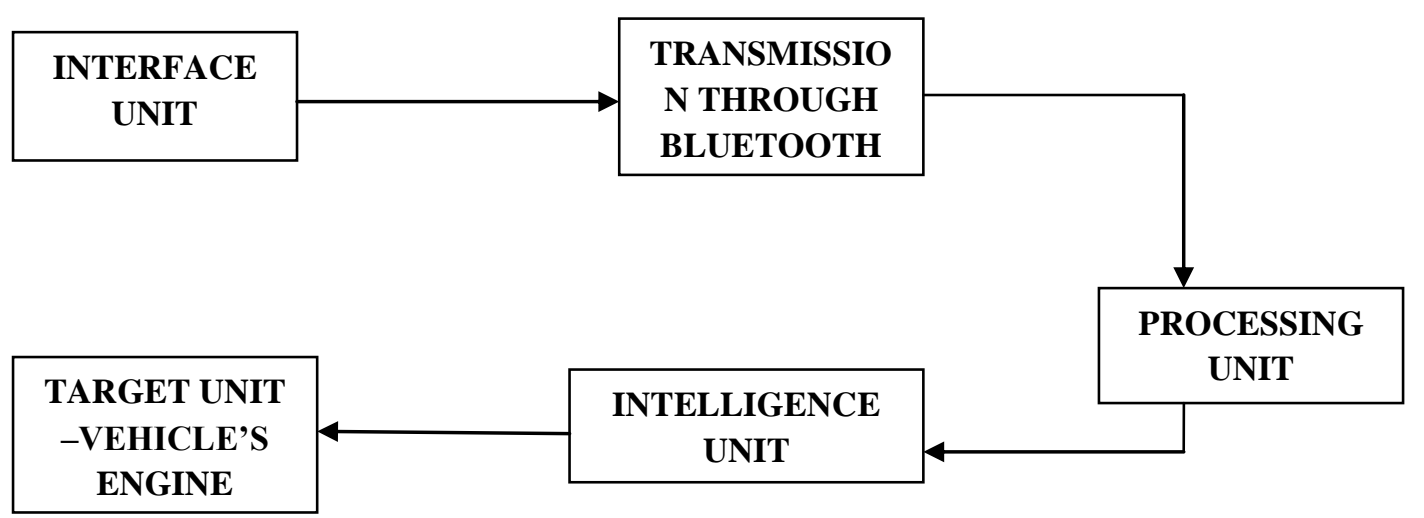

\subsection{Interface Unit}

This unit acquires EEG signals of the driver for the detection of alcohol. This unit has to ensure effective electrode system yet not irritate the users. "The recent research details the design, development and testing of a non-invasive mobile and wireless EEG system for continuously monitoring hightemporal resolution brain dynamics without requiring conductive gels applied to the scalp'.

A cap containing five embedded dry electrodes on the wearer's forehead and one electrode behind the left ear is proposed to be used to acquire EEG signals. Then, the EEG signals are wirelessly transmitted to a data receiver through Bluetooth, where they are processed in real-time.

\subsection{Digital Pre-Processing Unit}

This unit is a part of intelligent unit. The output from this digital pre processing unit is forced into the intelligent unit for further processing and decision making. It consists of preamplifiers and digital filters to remove the noise associated in the wave form such as power-line and base line interferences or noises. Preamplifiers are high input impedance amplifiers with high signal to noise ratio. This is in order to prevent mixing of noise while measuring EEG and to preserve the significant portions of the low frequency low amplitude biosignal. Buffers can also be integrated for temporary storage.

\subsection{Intelligence Unit}

Intelligence unit consists of a processor for processing the acquired EEG waves. This unit is placed in such a way that it enables or disables the engine depending upon absence or presence of EEG abnormalities.
Fig 12: Block diagram

\section{INTERFACING WITH ENGINE OF THE VEHICLE}

Batteries of $12 \mathrm{v}$ or $24 \mathrm{v}$ or $42 \mathrm{v}$ are generally employed in the automobiles for starting and for the functioning of the electrical systems. Whenever the driver intends to start his vehicle through his key, the ignition system circuit is completed. This allows the battery current to flow through the ignition coil which energizes the electronic spark distributor that is interconnected to the engine camshaft, which helps in providing the spark at appropriate time for efficient combustion of the charge. In the modern cars, an ECU determines the appropriate time by the inputs from numerous sensors.

A relay system replaces the key. Relay is driven by the voltage that is provided by the intelligent unit depending upon the presence or absence of abnormalities in the EEG.

Now if any abnormality is detected by the algorithm relay drivable voltage is not produced so battery of the vehicle and relay system stays open and engine doesn't start. And vice versa happens if abnormality is not detected.

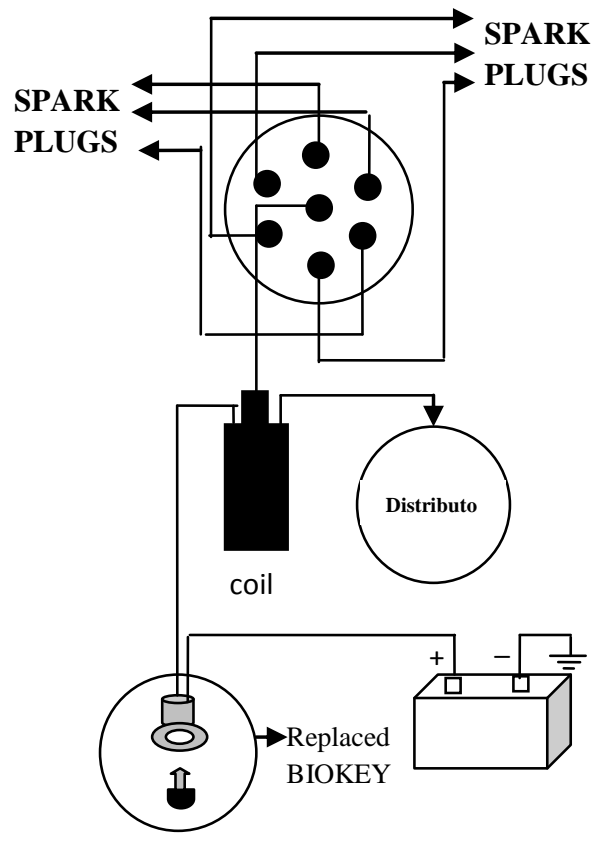

Fig 13: Engine interfaced with biokey 


\section{CONCLUSION}

The above results support the hypothesis that it is possible to prevent drivers from causing accidents due to drunken driving. Since this method deals with biological signals to identify the presence of alcohol, it is more efficient than the previous techniques. Moreover it is evident that power spectrum of EEG is affected by alcoholic intake. So, as and when the product actually enters the market as a standard equipment/accessory of the vehicle, it will surely yield much greater benefits of saving thousands of human lives on roads every year.

\section{REFERENCE}

[1] Philip, P. Vervialle, F. Le Breton, P. Taillard, J. Horne, J.A. 2001. Fatigue, alcohol, and serious road crashes in France: factorial study of national data. BMJ. 322:82930.

[2] Connor, J. Norton, R. Ameratunga, S., et al. 2002. Driver sleepiness and risk of serious injury to car occupants: population based case-control study. BMJ. 324:1125-8.

[3] Horne, J.A., Reyner, L.A.1995. Sleep related vehicle accidents. BMJ. 310,565-7.

[4] Banks, S. Catcheside, P. Lack, L. Grunstein, R.R. McEvoy, R.D. 2004. Low levels of alcohol impair driving simulator performance and reduce perception of crash risk in partially sleep deprived subjects. Sleep. 27, $1063-7$.

[5] AshkanYazdani, S. Kamaledin Setarehdan. Classification of EEG signal correlated with alcohol abusers. Control and Intelligent Processing Centre of Excellence, School of ECE, Faculty of Engineering, University of Tehran, Iran.ISBN-1-4244-0779-6/07-2007.

[6] Zhong, S. and Ghosh, J. 2002.HMMs and coupled HMMs for multichannel EEG classification. Proc. IEEE Int. Joint Conf. on Neural Networks, Honolulu, Hawaii.1154-1159.

[7] Welch, P.D. 1967.The Use of Fast Fourier Transform for the Estimation of Power Spectra: A Method Based on Time Averaging Over Short, Modified Periodogram. IEEE Trans. Audio \& Electroacoust.15, 70-73.

[8] Vadim, V. Nikulin, Anna, V. Nikulina, Hidehisa yamashita. 2005. Effects of alcohol on spontaneous neuronal oscillations: A combined magneto encephalography and electroencephalography study. Progress in Neuro-Psychopharmacology \& Biological Psychiatry. 19, 687-693.

[9] X. L. Zhang, H. Begleiter, B. Porjesz, and A. Litke. 1997. Electrophysiological evidence of memory Impairment in alcoholic patients. Biological Psychiatry. 42,1157-1171

[10] Ge Zhexue, Chen Zhongsheng. 2006. Application of Time-Frequency Analysis based on Matlab. Beijing: Posts \& Telecom Press.
[11] Su Jinming, Zhang Lianhua, Liu Bo, "The Application of MA TLAB Toolboxes," Beijing: Publishing House of Electronics Industry, 2004.

[12] S. V. Borisov, A. Ya. Kaplan, N. L. Gorbachevskaya, and I. A.Kozlova. 2005. Analysis of EEG Structural Synchrony in Adolescents with Schizophrenic Disorders. Human Physiology. 31, 255-261.

[13] Lukas, S.E. Mendelson J.H. Benedict, R.A. Jones, B 1986. EEG alpha activity increases during transient episodes of ethanol-induced euphoria. Pharmacol Biochem. Behav. 25, 889-895.

[14] M. Treisman.1984.Temporal rhythms and cerebral rhythms. In Timing and Time Perception, J. Gibbon and L. Allan, Eds. New York.423, 542-565.

[15] J. Beatty, A. Greenberg, W. P. Deibler, and J. O'Hanlon, 1974. Operant control of occipital theta rhythm affects performance, in a radar monitoring task. Science.183, 871-873.

[16] S. Makeig and M. Inlow.1993.Lapses in alertness: Coherence of fluctuations in performance and EEG spectrum. Electroencephalography Clinical Neurophysiology.86, 23-35.

[17] Rangaswamy, M. Porjesz, B. Chorlian, D.B. Wang, K. Jones, K.A. Bauer, L.O. Rohrbaugh, J. O'Connor, S.J. Kuperman, S. Reich, T. Begleiter, H. 2002.Beta power in the EEG of alcoholics. Biol. Psychiatry 52, 831-842.

[18] Engel, G.L. Rosenbaum, M. 1944. Studies of the elctroencephalogram in acute alcoholic intoxication. Proc. Cent. Soc. Clin. Res.17, 62-63.

[19] Davis, P.A. Gibbs, F.A. Davis, H. Jetter, W.W Trowbridge, I.S.1941. The effects of alcohol upon the electroencephalogram (brian waves).Q. J. Stud. Alcohol. $1,626-637$

[20] Ehlers, C.L. Wall, T.L. Schuckit, M.A.1989. EEG spectral characteristics following ethanol administration in young men. Electroencephalogr. Clin. Neurophysiol. $73,179-187$.

[21] Slawecki, C.J. Bentancourt, M. Li, T.K. Ehlers, C.L. 2000. Neurophysiological findings and drinking levels in high-alcohol-drinking (HAD) and low-alcohol-drinking (LAD) rats. Alcsm. Clin. Exp. Res. 24, 1492-1499.

[22] Pollock, V.E. Schneider, L. Lyness, S.1992. Reliability of topographic quantitative EEG amplitudes in healthy, late middle-aged and elderly adults Electroencephalography Clin. Neurophysiology. 79, 2026.

[23] Saletu, B. 1997.Visualizing the living human brian. The techniques and promise of EEG and event-related potentials mapping. Bibl. Psychiatr.167, 54-62. 